\title{
Decomposable Ternary Cubics
}

\author{
Jaydeep V. Chipalkatti
}

\section{CONTENTS}

1. Introduction

2. Preliminaries

3. The Locus $X_{\equiv}$

4. The Locus $X_{\neq}$

5. The Locus $X_{\Delta}$

6. The Locus $\boldsymbol{X}_{\varnothing}$

7. The Locus $X_{\mid}$

8. The Locus $X_{Y}$

9. Ideal Generators and Concomitants References

2000 AMS Subject Classification: Primary 14-04, 14L35; Secondary 14Q99

Keywords: invariant, covariant, minimal resolution
Cubic forms in three variables are parametrised by points of a projective space $\mathbb{P}^{9}$. We study the subvarieties in this space defined by decomposable forms. Specifically, we calculate their equivariant minimal resolutions and describe their ideals invariant-theoretically.

\section{INTRODUCTION}

Let $F$ be a nonzero cubic form in variables $x_{1}, x_{2}, x_{3}$, written as follows:

$$
F=a_{0} x_{1}^{3}+a_{1} x_{1}^{2} x_{2}+\cdots+a_{9} x_{3}^{3}, \quad a_{0}, \ldots, a_{9} \in \mathbf{C}
$$

We identify $F$ with the point $\left[a_{0}, \ldots, a_{9}\right] \in \mathbb{P}^{9}$ and denote the homogeneous coordinate ring of $\mathbb{P}^{9}$ with $\mathbf{C}\left[a_{0}, \ldots, a_{9}\right]$. Now consider the set of those $F$ which factor as a product of a quadratic and a linear form, i.e., let

$$
\begin{aligned}
& X_{\varnothing}=\left\{F \in \mathbb{P}^{9}:\right. \\
& F=Q . L \text { for some forms } Q, L \text { of degrees } 2,1\} .
\end{aligned}
$$

This is a projective subvariety of $\mathbb{P}^{9}$. The group $S L_{3}(\mathbf{C})$ acts on $\mathbb{P}^{9}$ as follows: given a matrix $A \in S L_{3}$, introduce new variables $\left[x_{1}^{\prime}, x_{2}^{\prime}, x_{3}^{\prime}\right]=\left[x_{1}, x_{2}, x_{3}\right] A$, and define $a_{0}^{\prime}, \ldots, a_{9}^{\prime}$ by forcing the identity

$$
\begin{aligned}
a_{0} x_{1}^{3}+a_{1} x_{1}^{2} x_{2}+\cdots+a_{9} x_{3}^{3}=a_{0}^{\prime} & x_{1}^{3}+a_{1}^{\prime} x_{1}^{\prime 2} x_{2}^{\prime} \\
& +\cdots+a_{9}^{\prime} x_{3}^{\prime 3} .
\end{aligned}
$$

Then $\left[a_{0}, \ldots, a_{9}\right] \stackrel{A}{\longrightarrow}\left[a_{0}^{\prime}, \ldots, a_{9}^{\prime}\right]$. The imbedding $X_{\varnothing} \subseteq \mathbb{P}^{9}$ is thus $S L_{3}(\mathbf{C})$-equivariant. Hence $I_{X_{\varnothing}}<$ $\mathbf{C}\left[a_{0}, \ldots, a_{9}\right]$ is a representation of $S L_{3}$, and so are all the syzygy modules in the minimal resolution of $I_{X_{\varnothing}}$.

There are different possible factorisations of $F$, leading to five similarly defined varieties:

$$
\begin{aligned}
& X_{\equiv}=\left\{F \in \mathbb{P}^{9}: F=L^{3} \text { for a linear form } L\right\} \\
& X_{\neq}=\left\{F \in \mathbb{P}^{9}: F=L_{1}^{2} L_{2} \text { for some } L_{i}\right\} \\
& X_{\Delta}=\left\{F \in \mathbb{P}^{9}: F=L_{1} L_{2} L_{3} \text { for some } L_{i}\right\}
\end{aligned}
$$


and the loci $X_{\triangleright}, X_{Y}$ defined as Zariski closures

$$
\begin{aligned}
X_{\mid \bigcirc}= & \left\{F \in \mathbb{P}^{9}: F=Q . L \text { where the conic } Q=0\right. \\
& \text { is smooth and tangent to the line } L=0\} \\
X_{Y}= & \left\{F \in \mathbb{P}^{9}: F=L_{1} L_{2} L_{3} \text { where } L_{i}=0\right. \\
& \text { are concurrent lines }\} .
\end{aligned}
$$

All the loci are irreducible, and there are inclusions

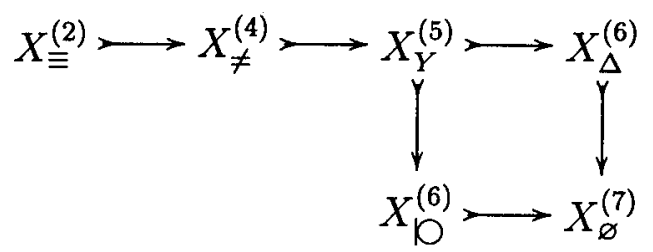

The superscripts indicate dimensions. Consider the following two problems:

(I) calculating the $S L_{3}$-equivariant minimal resolution for each variety $X$, and

(II) expressing the minimal set of generators of $I_{X}$ (i.e., the degree zero syzygies), in the language of classical invariant theory.

Problem II, in a slightly weaker form, is of classical origin. A priori, we are looking for a finite set of concomitants of $F$, such that their vanishing is a necessary and sufficient condition for $F$ to lie in $X$. Describing such a set is tantamount to describing $I_{X}$ up to its radical.

This is an instance of the general philosophy that for a form $F$ (of any degree in any number of variables), any property which is invariant under a linear change of variables (e.g., decomposabililty, existence of singular points, expressibility as a sum of a specified number of powers of linear forms) should be characterisable by the vanishing (or non-vanishing) of certain concomitants of $F$. Classical literature on invariant theory contains a very large number of results along these lines-see e.g. [Dolgachev and Kanev 93, Grace and Young 1903, Salmon 1876, Weitzenböck 1923] or the collected papers of Cayley and Clebsch. Salmon's book [Salmon 1879] is an excellent reference for the classical theory of plane curves and connections to invariant theory. Its chapter $\mathrm{V}$ is entirely devoted to ternary cubics. For newer (i.e. post-Hilbert) directions in invariant theory, see [Carrell and Dieudonné 71, Kraft 85, Weyl 1939].

Problem I is a natural generalisation of II, but certainly more modern. In this paper we solve problem I, and then interpret the representations corresponding to the ideal generators as concomitants of ternary cubics.

Some of our varieties have alternate descriptions, e.g., $X_{\equiv}$ is the cubic Veronese imbedding of $\mathbb{P}^{2}$ and $X_{Y}$ is a rank variety in the sense of Porras [Porras 96]. As such, the minimal systems of ideal generators for them are known. The standard equations defining the Veronese are given in [Harris 92, p. 23], but not in invarianttheoretic language. We will describe Porras's deduction of the minimal resolution of $X_{Y}$ in Section 8. In the book referred to above, Salmon deduces sets of equations describing the varieties $X_{\Delta}$ and $X_{\varnothing}$ set-theoretically. Our calculations confirm that these equations generate the ideal $I_{X}$ in each case.

The next section contains background material on representation theory. For each of the loci $X$, we find the ideal $I_{X}$ and its Betti numbers using a machine computation. Then we identify the syzygy modules as $S L_{3^{-}}$ representations using the hypercohomology spectral sequence, together with algebraic considerations specific to each $X$. In the last section, we write down the concomitants corresponding to the ideal generators.

This paper was inspired by the Weltanschauung of [Fulton and Harris 91] (especially $\S 11.3, \S 13.4$ ), and also by Salmon's wonderful book [Salmon 1879]. The program Macaulay-2 has been of immense help in the computations, and it is a pleasure to thank its authors Daniel Grayson and Michael Stillman. I thank the referee for helpful observations and Anthony V. Geramita, Leslie Roberts and the Queen's University for financial assistance during the course of this work.

\section{PRELIMINARIES}

The purpose of this section is to establish notation and state the working definitions, all of which are phrased in invariant-theoretic terms. I have written somewhat expansively, in the hope that the framework introduced here may be more generally applicable.

Let $V$ be a three dimensional $\mathbf{C}$-vector space. All representations considered henceforth are for the group $S L(V)$. (See [Fulton 97, Fulton and Harris 91, Sturmfels 93] for the theory.) For a pair $(m, n)$ of nonnegative integers, we have the Schur module $S_{m+n, n}=S_{m+n, n}(V)$, with the convention that $S_{m}=S_{m, 0}=\operatorname{Sym}^{m}(V)$ and $S_{1,1}=\wedge^{2} V$. Note the isomorphism ${ }^{1} S_{m+n, n}^{*}=S_{m+n, m}$ and the formula

$$
\operatorname{dim} S_{m+n, n}=\frac{1}{2}(m+1)(n+1)(m+n+2) .
$$

In the sequel, we frequently need to decompose the tensor product $S_{m_{1}+n_{1}, n_{1}} \otimes S_{m_{2}+n_{2}, n_{2}}$ and the plethysm $S_{\lambda}\left(S_{m+n, n}\right)$ as direct sums of irreducible representations.

\footnotetext{
${ }^{1}$ Henceforth ' $=$ ' means an isomorphism of $S L$-modules.
} 
The former is governed by the Littlewood-Richardson rule and the latter by formulae (A.5), (A.21) in [Fulton and Harris 91, appendix A]. This was programmed in Maple by the author. ${ }^{2}$

Let $\underline{x}=\left\{x_{1}, x_{2}, x_{3}\right\}$ be a basis of $V^{*}$. The identification $\wedge^{2} V^{*}=V$ gives a basis $\underline{u}=\left\{u_{1}, u_{2}, u_{3}\right\}$ of $V$, where

$$
u_{1}=x_{2} \wedge x_{3}, u_{2}=x_{3} \wedge x_{1}, u_{3}=x_{1} \wedge x_{2} .
$$

If $a_{r}$ appears as the coefficient of $x_{1}^{i_{1}} x_{2}^{i_{2}} x_{3}^{i_{3}}$ in (1-1), redefine $a_{r}$ to be $u_{1}^{i_{1}} u_{2}^{i_{2}} u_{3}^{i_{3}}$. Thus $\underline{a}=\left\{a_{0}, \ldots, a_{9}\right\}$ is a basis of $S_{3}$ and the form $F$ is recast as the canonical trace element

$$
\sum_{i_{1}+i_{2}+i_{3}=3} u_{1}^{i_{1}} u_{2}^{i_{2}} u_{3}^{i_{3}} \otimes x_{1}^{i_{1}} x_{2}^{i_{2}} x_{3}^{i_{3}} \quad \in S_{3} \otimes S_{3}^{*} .
$$

The ring $\mathbf{C}\left[a_{0}, \ldots, a_{9}\right]$ is identified with the symmetric algebra $R=\bigoplus_{\ell \geq 0} S_{\ell}\left(S_{3}\right)$, and we declare $\mathbb{P} S_{3}^{*}=\operatorname{Proj} R$ to be the ambient space for all loci under consideration.

\subsection{A Basis for $\mathrm{S}_{\mathbf{m}+\mathbf{n}, \mathbf{n}}$}

There is a canonical injection (see [p.233 ff, loc.cit.])

$$
S_{m+n, n} \longrightarrow \operatorname{Sym}^{n}\left(\wedge^{2} V\right) \otimes \operatorname{Sym}^{m}(V),
$$

and the right hand side has a basis of monomials

$$
\left\{x_{1}^{n_{1}} x_{2}^{n_{2}} x_{3}^{n_{3}} \otimes u_{1}^{m_{1}} u_{2}^{m_{2}} u_{3}^{m_{3}}: \Sigma n_{i}=n, \Sigma m_{i}=m\right\} .
$$

Let $T$ be a semistandard tableau on numbers $1,2,3$ on the Young diagram of $(m+n, n)$. Say the entries in the first (resp. second) row are $r_{1} \leq \cdots \leq r_{n} \leq s_{1} \leq$ $\cdots \leq s_{m}$ (resp. $\left.t_{1} \leq \cdots \leq t_{n}\right)$, with $r_{i}<t_{i}$ for $1 \leq$ $i \leq n$. Let $x_{\left\langle r_{i}, t_{i}\right\rangle}$ denote resp. $x_{1},-x_{2}, x_{3}$ if $\left(r_{i}, t_{i}\right)=$ $(2,3),(1,3),(1,2)$, and write

$$
X_{T}=\prod_{i=1}^{n} x_{\left\langle r_{i}, t_{i}\right\rangle} \otimes \prod_{i=1}^{m} u_{s_{i}}
$$

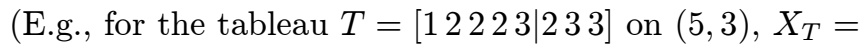
$x_{1}^{2} x_{3} \otimes u_{2} u_{3}$.) Now (ignoring signs) the subset $\left\{X_{T}\right.$ : $T$ semistandard $\}$ of $(2-3)$ is a basis for $S_{m+n, n}$.

\subsection{Concomitants}

Let us assume that we have an injective map of representations

$$
S_{m+n, n} \stackrel{\varphi}{\longrightarrow} S_{\ell}\left(S_{3}\right),
$$

\footnotetext{
${ }^{2}$ The 'Symmetrica' package and John Stembridge's 'SF' package do this too, but I have found that ad-hoc routines for $\operatorname{dim} V=3$ tend to work faster. Of course the issue of speed is more serious for the plethysm.
}

or equivalently, an imbedding

$$
\mathbf{C} \longrightarrow S_{\ell}\left(S_{3}\right) \otimes S_{m+n, n}^{*}\left(=S_{\ell}\left(S_{3}\right) \otimes S_{m+n, m}\right) .
$$

Let $\Phi$ denote the image of 1 , which is a $\mathbf{Q}$-linear combination of monomials

$$
\left\{\prod_{i=0}^{9} a_{i}^{l_{i}} \otimes \prod_{i=1}^{3} x_{i}^{m_{i}} \otimes \prod_{i=1}^{3} u_{i}^{n_{i}}: \Sigma l_{i}=\ell, \Sigma m_{i}=m, \Sigma n_{i}=n\right\} .
$$

In 19th century terminology (due to Sylvester), $\Phi$ is called a concomitant of degree $\ell$, order $m$ and class $n$ (for ternary cubics). We indicate this by writing $\Phi_{\ell, m, n}$. Of course, there may exist more than one concomitant for given $(\ell, m, n)$ (or none at all). A concomitant is called a covariant (resp. contravariant) if its class (resp. order) is zero, and an invariant if both are zero. Note that the imbedding $\varphi$ can be recovered from $\Phi$.

For instance, the Hessian of a ternary cubic is a covariant of degree 3 and order 3 . The dual of the curve $F=0$ is defined by a contravariant of degree 4 and class 6 .

\subsection{The Symbolic Method}

We will represent concomitants using the German symbolic method. A brief explanation follows, but it is unlikely to be intelligible without prior acquaintance with the method. The notation follows Grace and Young [Grace and Young 1903], also see [Olver 99, Ch. 6] for a modern exposition.

We will use the letters $x_{1}, x_{2}, x_{3} ; u_{1}, u_{2}, u_{3}$. In addition, we have an indefinite supply of indexed Greek letters $\alpha_{1}, \alpha_{2}, \alpha_{3} ; \beta_{1}, \beta_{2}, \beta_{3}$ etc. Set

$$
\alpha_{x}=\alpha_{1} x_{1}+\alpha_{2} x_{2}+\alpha_{3} x_{3} \text { and ditto for } \beta_{x}, \gamma_{x} \text { etc. }
$$

Formally write $F=\alpha_{x}^{3}=\beta_{x}^{3}=\ldots$ That is to say, after expansion, $\frac{3 !}{i_{1} ! i_{2} ! i_{3} !} \alpha_{1}^{i_{1}} \alpha_{2}^{i_{2}} \alpha_{3}^{i_{3}}$ stands for the appropriate coefficient $a_{r}$ in (1-1). The symbols $(\alpha \beta \gamma),(\alpha \beta u)$ respectively stand for the determinants

$$
\left|\begin{array}{lll}
\alpha_{1} & \alpha_{2} & \alpha_{3} \\
\beta_{1} & \beta_{2} & \beta_{3} \\
\gamma_{1} & \gamma_{2} & \gamma_{3}
\end{array}\right| \text { and }\left|\begin{array}{ccc}
\alpha_{1} & \alpha_{2} & \alpha_{3} \\
\beta_{1} & \beta_{2} & \beta_{3} \\
u_{1} & u_{2} & u_{3}
\end{array}\right|
$$

Now for instance, the Hessian is written $(\alpha \beta \gamma)^{2} \alpha_{x} \beta_{x} \gamma_{x}$. To evaluate this, multiply out the expression formally, and substitute the $a_{r}$ 's as above. The result, appropriately, is a polynomial of degree 3 each in the $\underline{a}, \underline{x}$. As another example, the dual curve mentioned above has equation $(\alpha \beta u)^{2}(\gamma \delta u)^{2}(\alpha \gamma u)(\beta \delta u)=0$.

Each concomitant $\Phi_{\ell, m, n}$ has a (non-unique) expression as a sum of products of symbols of the form $\alpha_{x},(\alpha \beta \gamma),(\alpha \beta u)$. In each summand, the letters $x, u$ 
should occur resp. $m, n$ times, along with $\ell$ Greek letters, each occuring exactly thrice. Conversely, every such symbolic expression corresponds to a concomitant, unless it vanishes identically after substitution.

\subsection{A Spectral Sequence}

Let $X$ be any of the loci above. The equivariant minimal resolution of its defining ideal $I_{X}<R$, will be written

$$
\ldots \rightarrow F^{p} \rightarrow F^{p+1} \rightarrow \ldots \rightarrow F^{0} \rightarrow I_{X} \rightarrow 0,
$$

with $\quad F^{p}=\bigoplus_{j>0} M_{j,-p} \otimes R(-j+p) \quad$ for $p \leq 0$.

Letting $\mathcal{F}^{p}=\left(F^{p}\right)=\bigoplus M_{j,-p} \otimes \mathcal{O}_{\mathbb{P}^{9}}(-j+p)$, we have a resolution $\mathcal{F}^{\bullet} \rightarrow \mathcal{I}_{X} \rightarrow 0$, for the ideal sheaf. For $\ell \in \mathbf{Z}$, let $\mathcal{F}^{p}(\ell)=\mathcal{F}^{p} \otimes \mathcal{O}_{\mathbb{P}}(\ell)$. Then we have a second quadrant spectral sequence

$$
\begin{aligned}
& \check{E}_{1}^{p, q}=H^{q}\left(\mathbb{P}^{9}, \mathcal{F}^{p}(\ell)\right) \quad \text { for } p \leq 0, q \geq 0, \\
& d_{r}: \check{E}_{r}^{p, q} \rightarrow \check{E}_{r}^{p+r, q-r+1} ; \quad \check{E}_{\infty}^{p, q} \Rightarrow H^{p+q}\left(\mathbb{P}^{9}, \mathcal{I}_{X}(\ell)\right) .
\end{aligned}
$$

This will be used in conjunction with the standard calculation of cohomology of line bundles on projective space ([Hartshorne 77, Ch. III, Theorem 5.1]) and Serre duality.

The bulk of the paper is concerned with the identification of the $M_{j,-p}$ qua $S L_{3}-$ representations. The ideals and their Betti numbers (i.e. dimensions of $M_{j,-p}$ ) were calculated in Macaulay-2.

\subsection{Computation of $I_{X}$}

For illustration, consider the locus $X_{\neq}$. It is the image of the projective morphism

$$
\mathbb{P} V^{*} \times \mathbb{P} V^{*} \longrightarrow \mathbb{P} S_{3}^{*}, \quad\left(L_{1}, L_{2}\right) \rightarrow L_{1}^{2} L_{2} .
$$

Let $L_{1}=b_{1} x_{1}+b_{2} x_{2}+b_{3} x_{3}, \quad L_{2}=c_{1} x_{1}+c_{2} x_{2}+c_{3} x_{3}$, where the $b_{i}, c_{i}$ are indeterminates. By forcing the equality $F=L_{1}^{2} L_{2}$, we get polynomial expressions

$$
a_{r}=\varphi_{r}\left(b_{1}, b_{2}, b_{3} ; c_{1}, c_{2}, c_{3}\right) \text { for } r=0, \ldots, 9 ;
$$

defining a ring map $f_{\neq}: \mathbf{C}\left[a_{0}, \ldots, a_{9}\right] \longrightarrow \mathbf{C}\left[b_{1}, \ldots, c_{3}\right]$. Then $I_{X_{\neq}}$equals $\operatorname{ker}\left(f_{\neq}\right)$. Its minimal resolution is tabulated as follows:

\begin{tabular}{c|ccccccc} 
& 0 & 1 & 2 & 3 & 4 & 5 & 6 \\
\hline 3 & 20 & 45 & 36 & 10 & & & \\
4 & 28 & 126 & 225 & 200 & 90 & 18 & 1
\end{tabular}

In the notation of $(2-5)$, the entry in row $j$ and column $-p$ is $\operatorname{dim} M_{j,-p}$. E.g., $M_{3,2}$ is 36 -dimensional.
The loci $X_{\equiv}, X_{\Delta}, X_{\varnothing}$ are images of projective morphisms, respectively from $\mathbb{P} V^{*},\left(\mathbb{P} V^{*}\right)^{3}, \mathbb{P} S_{2}^{*} \times \mathbb{P} V^{*}$ to $\mathbb{P} S_{3}^{*}$; hence a similar procedure works for them. That these loci are closed, follows from the fundamental theorem of elimination theory.

In order to find $I_{X_{1}}$, we need the tact-invariant of a conic and a line (see [Salmon 1879, §96]). Let

$$
\begin{aligned}
Q & =q_{1} x_{1}^{2}+q_{2} x_{2}^{2}+q_{3} x_{1} x_{2}+q_{4} x_{1}+q_{5} x_{2}+q_{6}, \\
L & =b_{1} x_{1}+b_{2} x_{2}+b_{3} .
\end{aligned}
$$

Writing $Q . L=\left.F\right|_{\left(x_{3}=1\right)}$, we get expressions $a_{r}=$ $\varphi_{r}\left(q_{1}, \ldots, b_{3}\right)$ as before. Let $\operatorname{Res}=\operatorname{Resultant}\left(Q, L ; x_{2}\right)$, then the $x_{1}$-values for which $\operatorname{Res}=0$ are the $x_{1}$ coordinates of the points $Q=L=0$. The condition that they coincide is $T^{\prime}=$ Discriminant $\left(\operatorname{Res}, x_{1}\right)=0$. Then the tact-invariant $T=\frac{1}{b_{2}^{2}} T^{\prime}$. It vanishes iff the line $L=0$ is tangent to $Q=0$. (The extraneous factor appears because when $b_{2}=0$, the $x_{1}$-coordinates coincide for any position of $Q$.) For what it is worth,

$$
\begin{aligned}
T= & 4 q_{2} q_{6} b_{1}^{2}-4 q_{2} q_{4} b_{1} b_{3}+4 q_{1} q_{2} b_{3}^{2}-q_{5}^{2} b_{1}^{2}-4 q_{3} q_{6} b_{1} b_{2} \\
& +2 q_{4} q_{5} b_{1} b_{2}+2 q_{3} q_{5} b_{1} b_{3}-q_{4}^{2} b_{2}^{2}-4 q_{1} q_{5} b_{2} b_{3} \\
& +2 q_{3} q_{4} b_{2} b_{3}-q_{3}^{2} b_{3}^{2}+4 q_{1} q_{6} b_{2}^{2} .
\end{aligned}
$$

Now $I_{X_{1}}$ is the kernel of the map

$$
f_{\mid \mathrm{O}}: \mathbf{C}\left[a_{0}, \ldots, a_{9}\right] \longrightarrow \mathbf{C}\left[q_{1}, \ldots, b_{3}\right] /(T) .
$$

To calculate $I_{X_{Y}}$, let

$$
\begin{aligned}
& L_{1}=b_{1} x_{1}+b_{2} x_{2}+b_{3} x_{3} \\
& L_{2}=c_{1} x_{1}+c_{2} x_{2}+c_{3} x_{3} \\
& L_{3}=d_{1} x_{1}+d_{2} x_{2}+d_{3} x_{3}
\end{aligned} \text { and } D=\left|\begin{array}{ccc}
b_{1} & b_{2} & b_{3} \\
c_{1} & c_{2} & c_{3} \\
d_{1} & d_{2} & d_{3}
\end{array}\right| \text {. }
$$

Then $D=0$ is the condition that the lines be concurrent. The $\varphi_{r}$ are given by $F=L_{1} L_{2} L_{3}$, and $I_{X_{Y}}$ is the kernel of

$$
f_{Y}: \mathbf{C}\left[a_{0}, \ldots, a_{9}\right] \longrightarrow \mathbf{C}\left[b_{1}, \ldots, d_{3}\right] /(D) .
$$

\subsection{Characters}

We write the formal character of $S_{a, b}$ as $\left[S_{a, b}\right]=\sigma_{a, b}$. Every finite dimensional $S L_{3}$-module $U$ is uniquely expressible as a direct sum $\bigoplus\left(S_{a_{i}, b_{i}}\right)^{\oplus n_{i}}$, hence write $[U]=$ $\sum n_{i} \sigma_{a_{i}, b_{i}}$.

If $C^{\bullet}=\left\{C^{p}\right\}_{p}$ is a bounded complex of finite dimensional $S L_{3}-$ representations and $S L$-equivariant differentials, then define $\left[C^{\bullet}\right]=\sum_{p}(-1)^{p}\left[C^{p}\right]$. By Schur's lemma, we have

$$
\left[C^{\bullet}\right]=\sum_{p}(-1)^{p}\left[H^{p}\left(C^{\bullet}\right)\right] .
$$




\section{THE LOCUS $X_{\equiv}$}

The Betti numbers of $I_{X}$ are

\begin{tabular}{c|ccccccc} 
& 0 & 1 & 2 & 3 & 4 & 5 & 6 \\
\hline 2 & 27 & 105 & 189 & 189 & 105 & 27 & \\
3 & & & & & & & 1
\end{tabular}

Now $X$ is the cubic Veronese imbedding of $\mathbb{P}^{2}$, hence arithmetically Cohen-Macaulay. Since $\omega_{X}=\mathcal{O}_{X}(-1)$, it is moreover subcanonical, hence arithmetically Gorenstein. Thus the minimal resolution of $R / I_{X}$ is centrally symmetric, i.e., $M_{23}, M_{24}, M_{25}{ }^{3}$ are respectively dual to $M_{22}, M_{21}, M_{20}$.

Consider the diagram

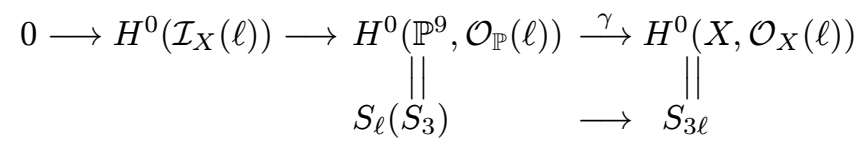

The map $\gamma$ is surjective for $\ell \geq 0$, hence by $(2-7)$,

$$
\left[H^{0}\left(\mathcal{I}_{X}(\ell)\right)\right]=\left[H^{0}\left(\mathcal{O}_{\mathbb{P}}(\ell)\right)\right]-\left[H^{0}\left(\mathcal{O}_{X}(\ell)\right)\right] .
$$

Substitute $\ell=2,3$, and decompose the plethysms involved, then

$$
\left[H^{0}\left(\mathcal{I}_{X}(2)\right)\right]=\sigma_{42}, \quad\left[H^{0}\left(\mathcal{I}_{X}(3)\right)\right]=\sigma_{72}+\sigma_{63}+\sigma_{33}+\sigma_{30}{ }^{4}
$$

Now successively let $\ell=2,3$ in the spectral sequence (2-6). In each case, all nonzero terms are in the row $q=0$, so $\check{E}_{2}=\check{E}_{\infty}$. Thus we have $M_{20}=H^{0}\left(\mathcal{I}_{X}(2)\right)$ and an exact sequence

$$
0 \longrightarrow M_{21} \longrightarrow M_{20} \otimes S_{3} \longrightarrow H^{0}\left(\mathcal{I}_{X}(3)\right) \longrightarrow 0 .
$$

It follows that $M_{20}=S_{42}$ and $M_{21}=S_{54} \oplus S_{51} \oplus S_{42} \oplus S_{21}$; hereafter written $M_{21}=\{54,51,42,21\}$. Now $M_{22}$ is calculated similarly using $\ell=4$. This is the end result:

$$
\begin{aligned}
& M_{20}=\{42\}, \\
& M_{21}=\{54,51,42,21\}, \\
& M_{22}=\{63,54,51,42,33,30,21\},
\end{aligned}
$$

and since these are all self-dual, $M_{25}=M_{20}, M_{24}=$ $M_{21}, M_{23}=M_{22}$ and of course $M_{36}=\{00\}$.

Problem 3.1. All the syzygy modules in the resolution are self-dual. One would like to know if this has any geometric significance and to what extent this is true of other Veronese imbeddings. (This is trivially true of the rational normal curve, since all $S L_{2}$-representations are

\footnotetext{
${ }^{3}$ Henceforth the commas are left out.

$4 \ldots$ and even here.
}

self-dual. But it fails for the quadratic imbedding of $\mathbb{P}^{2}$ in $\mathbb{P}^{5}$.)

As far as I know, the problem of describing an equivariant minimal resolution for the Veronese variety is open in general. See [Green 84] for some results.

\section{THE LOCUS $X_{\neq}$}

The Betti numbers of $I_{X}$ are

\begin{tabular}{c|ccccccc} 
& 0 & 1 & 2 & 3 & 4 & 5 & 6 \\
\hline 3 & 20 & 45 & 36 & 10 & & & \\
4 & 28 & 126 & 225 & 200 & 90 & 18 & 1
\end{tabular}

We have an exact sequence

$$
0 \longrightarrow \mathcal{I}_{X_{\neq}} \longrightarrow \mathcal{O}_{\mathbb{P}^{9}} \longrightarrow \mathcal{O}_{X_{\neq}} \longrightarrow 0,
$$

and to use the spectral sequence (2-6), some knowledge of the groups $H^{\bullet}\left(\mathbb{P}^{9}, \mathcal{I}_{X}(\ell)\right)$ is needed. We proceed in several steps.

\subsection{Step I}

Consider the commutative triangle

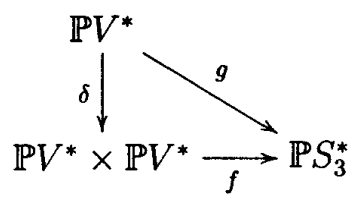

where $f:\left(L_{1}, L_{2}\right) \rightarrow L_{1}^{2} L_{2}$, and $\delta$ is the diagonal imbedding. Then image $f=X_{\neq}$, image $g=X_{\equiv}$. Define an

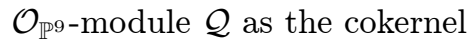

$$
0 \longrightarrow \mathcal{O}_{X_{\neq}} \longrightarrow f_{*} \mathcal{O}_{\mathbb{P}^{2} \times \mathbb{P}^{2}} \longrightarrow \mathcal{Q} \longrightarrow 0
$$

then $\operatorname{supp}(\mathcal{Q})=X_{\equiv}$.

Lemma 4.1. There is an isomorphism $g^{*} \mathcal{Q}=\Omega_{\mathbb{P}^{2}}^{1}$.

Proof: Let $\mathcal{J}$ be the ideal sheaf of image $(\delta)$, thus

$$
0 \longrightarrow \mathcal{J} \longrightarrow \mathcal{O}_{\mathbb{P}^{2} \times \mathbb{P}^{2}} \longrightarrow \delta_{*} \mathcal{O}_{\mathbb{P}^{2}} \longrightarrow 0
$$

We have a commutative ladder

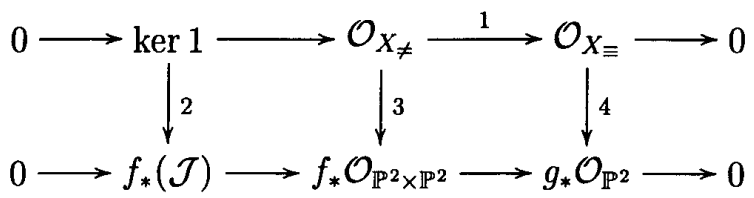

(Since $f$ is a finite morphism, $f_{*}$ is exact.) Since 4 is an isomorphism, coker $2=$ coker 3 , giving a surjection 
$f_{*}(\mathcal{J}) \stackrel{q}{\rightarrow} \mathcal{Q}$. By $([$ Hartshorne 77, p.110]), we have an adjoint morphism $\mathcal{J} \stackrel{q^{\prime}}{\longrightarrow} f^{*} \mathcal{Q}$. Now the composite

$$
f^{*} f_{*}(\mathcal{J}) \longrightarrow \mathcal{J} \stackrel{q^{\prime}}{\longrightarrow} f^{*} \mathcal{Q}
$$

is surjective, since $f^{*}$ is right-exact. Hence $q^{\prime}$ must be surjective. Applying $\delta^{*}$, we get a surjection $\delta^{*}(\mathcal{J}) \stackrel{q^{\prime \prime}}{\rightarrow}$ $g^{*} \mathcal{Q}$. By definition, $\Omega_{\mathbb{P}^{2}}^{1}$ equals $\delta^{*}(\mathcal{J})$.

To show that $q^{\prime \prime}$ is an isomorphism, it suffices to observe that both sheaves have the same Hilbert polynomial. By the Künneth formula,

$$
H^{0}\left(f_{*} \mathcal{O}_{\mathbb{P}^{2} \times \mathbb{P}^{2}} \otimes \mathcal{O}_{\mathbb{P}^{9}}(\ell)\right)=H^{0}\left(\mathbb{P}^{2}, \mathcal{O}_{\mathbb{P}}(2 \ell)\right) \otimes H^{0}\left(\mathbb{P}^{2}, \mathcal{O}_{\mathbb{P}}(\ell)\right)
$$

and this combined with $(4-1),(4-3)$ gives $9 \ell^{2}-1$ as the Hilbert polynomial of $\mathcal{Q}$. As $g^{*} \mathcal{O}_{\mathbb{P}^{9}}(\ell)=\mathcal{O}_{\mathbb{P}^{2}}(3 \ell)$, that of $g^{*} \mathcal{Q}$ is $\ell^{2}-1$. That this is also the Hilbert polynomial of $\Omega_{\mathbb{P}^{2}}^{1}$, can be seen from the Euler sequence. The lemma is proved.

\subsection{Step II}

The next step is to construct a complex of $S L_{3}$-modules with explicitly computable terms, such that the global sections of $\mathcal{I}_{X}(\ell)$ and $\mathcal{Q}(\ell)$ appear as its cohomology modules. We will use the Borel-Weil-Bott theorem several times (see e.g. [Chipalkatti 00, Theorem 0.1]).

Let $Y$ be the variety $\mathbb{P} V^{*} \times \mathbb{P} V^{*} \times \mathbb{P} S_{3}^{*}$ with projections $\mu_{1}, \mu_{2}, \pi$ onto the successive factors. Consider the diagram

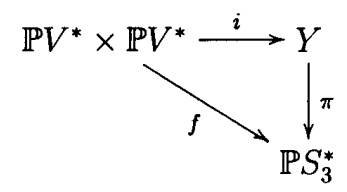

$$
\left(L_{1}, L_{2}\right) \stackrel{i}{\longrightarrow}\left(L_{1}, L_{2}, L_{1}^{2} L_{2}\right)
$$

and let $\Gamma \subseteq Y$ be the image of $i$. Then $\pi(\Gamma)=X_{\neq}$ (henceforth written $X$ ). We will derive a Koszul resolution of $\mathcal{O}_{\Gamma}$. Define a vector bundle

$$
\mathcal{A}=\mu_{1}^{*} \mathcal{O}_{\mathbb{P}^{2}}(2) \otimes \mu_{2}^{*} \mathcal{O}_{\mathbb{P}^{2}}(1) \otimes \pi^{*} T_{\mathbb{P}^{9}}(-1)
$$

on $Y$. If $y=\left(L_{1}, L_{2}, F\right)$ be a point of $Y$, then the fibre of $\mathcal{A}$ over $y$ is the 9 -dimensional vector space

$$
\left\langle L_{1}^{2}\right\rangle^{*} \otimes\left\langle L_{2}\right\rangle^{*} \otimes S_{3}^{*} /\langle F\rangle
$$

Here $\left\langle L_{1}^{2}\right\rangle \subseteq S_{2}^{*}$ is the one dimensional space generated by $L_{1}^{2}$, etc.

By the Künneth formula, $H^{0}(Y, \mathcal{A})=S_{2} \otimes S_{1} \otimes S_{3}^{*}$. The multiplication map $S_{2}^{*} \otimes S_{1}^{*} \rightarrow S_{3}^{*}$ corresponds to a distinguished section $\xi$ in $H^{0}(Y, \mathcal{A})$.

Lemma 4.2. The zero scheme of $\xi$ is $\Gamma$.
Proof: To say that $\xi$ vanishes at $y$, is to say that the element $L_{1}^{2} L_{2}+\langle F\rangle \in S_{3}^{*} /\langle F\rangle$ is zero. This happens iff $y \in \Gamma$.

Now $\operatorname{codim}(\Gamma, Y)=\operatorname{rank} \mathcal{A}=9$, hence the Koszul complex of $\xi$ resolves $\Gamma$.

$$
\begin{aligned}
0 \rightarrow \wedge^{9} \mathcal{A}^{*} & \rightarrow \cdots \wedge^{-p} \mathcal{A}^{*} \stackrel{\wedge \xi}{\rightarrow} \wedge^{-(p+1)} \mathcal{A}^{*} \\
& \rightarrow \ldots \mathcal{O}_{Y} \rightarrow \mathcal{O}_{\Gamma} \rightarrow 0, \text { for }-9 \leq p \leq 0
\end{aligned}
$$

Consider the hypercohomology spectral sequence

$$
\begin{array}{ll}
E_{1}^{p, q}=\mathbf{R}^{q} \pi_{*}\left(\wedge^{-p} \mathcal{A}^{*}\right), & d_{r}: E_{r}^{p, q} \longrightarrow E_{r}^{p+r, q-r+1} \\
E_{\infty}^{p+q} \Rightarrow \mathbf{R}^{p+q} \pi_{*}\left(\mathcal{O}_{\Gamma}\right) & \text { for }-9 \leq p \leq 0, q \geq 0
\end{array}
$$

Now

$$
\wedge^{-p} \mathcal{A}^{*}=\mu_{1}^{*} \mathcal{O}_{\mathbb{P}^{2}}(2 p) \otimes \mu_{2}^{*} \mathcal{O}_{\mathbb{P}^{2}}(p) \otimes \pi^{*} \Omega_{\mathbb{P}^{9}}^{-p}(-p)
$$

hence

$$
\begin{aligned}
& \mathbf{R}^{q} \pi_{*}\left(\wedge^{-p} \mathcal{A}^{*}\right) \\
& \quad=\left\{\bigoplus_{i+j=q} H^{i}\left(\mathbb{P}^{2}, \mathcal{O}_{\mathbb{P}^{2}}(2 p)\right) \otimes H^{j}\left(\mathbb{P}^{2}, \mathcal{O}_{\mathbb{P}^{2}}(p)\right)\right\} \otimes \Omega_{\mathbb{P}^{9}}^{-p}(-p) .
\end{aligned}
$$

Apart from $E_{1}^{0,0}=\mathcal{O}_{\mathbb{P}^{9}}$, all nonzero terms are concentrated in the row $q=4$ and columns $-9 \leq p \leq-3$. Using Serre duality,

$$
\begin{aligned}
E_{1}^{p, 4} & =H^{2}\left(\mathbb{P}^{2}, \mathcal{O}_{\mathbb{P}^{2}}(2 p)\right) \otimes H^{2}\left(\mathbb{P}^{2}, \mathcal{O}_{\mathbb{P}^{2}}(p)\right) \otimes \Omega^{-p}(-p) \\
& =S_{-(2 p+3)}^{*} \otimes S_{-(p+3)}^{*} \otimes \Omega^{-p}(-p) .
\end{aligned}
$$

Define a complex $\mathcal{G}^{\bullet}$ by $\mathcal{G}^{p}=E_{1}^{p, 4}$, with $d_{1}$ as the differential. It lives in the range $-9 \leq p \leq-3$.

Proposition 4.3. The cohomology of $\mathcal{G}^{\bullet}$ equals

$$
\mathcal{H}^{p}\left(\mathcal{G}^{\bullet}\right)\left(=E_{2}^{p, 4}\right)= \begin{cases}\mathcal{I}_{X} & \text { if } p=-5 \\ \mathcal{Q} & \text { if } p=-4 \\ 0 & \text { otherwise }\end{cases}
$$

Proof: Clearly $E_{2}=\cdots=E_{5}$ and $E_{6}=E_{\infty}$. Since $\left.\pi\right|_{\Gamma}$ is a finite morphism, $\mathbf{R}^{\geq 1} \pi_{*} \mathcal{O}_{\Gamma}=0$. Hence for $p \neq-5,-4$, we have $E_{2}^{p, 4}=E_{\infty}^{p, 4}=0$. Consider the extension

$$
0 \rightarrow E_{6}^{0,0} \rightarrow \pi_{*} \mathcal{O}_{\Gamma} \rightarrow E_{6}^{-4,4} \rightarrow 0 .
$$

The term $E_{6}^{0,0}$ is the image of the natural map $E_{1}^{0,0}$ (= $\left.\mathcal{O}_{\mathbb{P}^{9}}\right) \rightarrow \pi_{*} \mathcal{O}_{\Gamma}$, which is $\mathcal{O}_{X}$. Hence

$$
\begin{aligned}
& E_{5}^{-5,4}=\operatorname{ker}\left(\mathcal{O}_{\mathbb{P}^{9}} \rightarrow \mathcal{O}_{X}\right) \\
& E_{5}^{-4,4}=\operatorname{coker}\left(\mathcal{O}_{X} \rightarrow \pi_{*} \mathcal{O}_{\Gamma}\right) \quad=\mathcal{Q}
\end{aligned}
$$


For $\ell \in \mathbf{Z}$, write $\mathcal{G}^{\bullet}(\ell)=\mathcal{G}^{\bullet} \otimes \mathcal{O}_{\mathbb{P}^{9}}(\ell)$. All further calculation revolves around the hypercohomology of $\mathcal{G}^{\bullet}(\ell)$. There are two second quadrant spectral sequences

$$
\begin{aligned}
E_{1}^{p, q} & =H^{q}\left(\mathbb{P}^{9}, \mathcal{G}^{p}(\ell)\right), & & d_{r}: E_{r}^{p, q} \longrightarrow E_{r}^{p+r, q-r+1} ; \\
{ }^{\prime} E_{2}^{p, q} & =H^{q}\left(\mathbb{P}^{9}, \mathcal{H}^{p}\left(\mathcal{G}^{\bullet}(\ell)\right)\right), & & { }^{\prime} d_{r}:{ }^{\prime} E_{r}^{p, q} \longrightarrow{ }^{\prime} E_{r}^{p-r+1, q+r} ;
\end{aligned}
$$

with $E_{\infty}^{p, q},{ }^{\prime} E_{\infty}^{p, q} \Rightarrow \mathbb{H}^{p+q}\left(\mathcal{G}^{\bullet}(\ell)\right)$. If $\ell$ is clear from the context, we write $\mathbb{H}^{j}$ for $\mathbb{H}^{j}\left(\mathcal{G}^{\bullet}(\ell)\right)$. Henceforth a symbol such as $E_{r}^{p, q}$ refers to (4-7), and not to (4-5).

\subsection{Step III: Analysis of Equation (4-7)}

The terms

$$
E_{1}^{p, q}=S_{-(2 p+3)}^{*} \otimes S_{-(p+3)}^{*} \otimes H^{q}\left(\mathbb{P}^{9}, \Omega_{\mathbb{P}^{9}}^{-p}(\ell-p)\right)
$$

and ${ }^{\prime} E_{2}^{-4, q}=H^{q}\left(\mathbb{P}^{9}, \mathcal{Q}(\ell)\right)$ are evaluated by appealing to the Borel-Weil-Bott theorem. The result is as follows:

1. Assume $\ell>0$, and let $\lambda=(\ell, \underbrace{1, \ldots, 1}_{-p \text { times }})$. Then

$$
H^{q}\left(\Omega^{-p}(\ell-p)\right)= \begin{cases}S_{\lambda}\left(S_{3}\right) & \text { for } q=0, \\ 0 & \text { for } q \neq 0 .\end{cases}
$$

Moreover $H^{q}(\mathcal{Q}(\ell))$ is $S_{3 \ell-1,1}$ for $q=0$ and 0 if $q \neq 0$.

2. Assume $-9 \leq \ell \leq-1$. Then

$$
H^{q}\left(\Omega^{-p}(\ell-p)\right)= \begin{cases}\mathbb{C} & \text { for }(p, q)=(\ell,-\ell) \\ 0 & \text { otherwise }\end{cases}
$$

Moreover $H^{q}(\mathcal{Q}(\ell))$ is $S_{-(3 \ell+1),-(3 \ell+2)}$ if $q=2$ and 0 if $q \neq 2$.

Other values of $\ell$ are not difficult to analyse, but we will not need them. One can now decompose (4-9) into irreducible pieces using plethysms and the LittlewoodRichardson rule.

\subsection{Step IV: Analysis of Equation (4-8) and $\mathbb{H}^{\bullet}$}

The term ' $E_{2}^{p, q}$ can be nonzero only for $p=-5,-4$, so ${ }^{\prime} E_{3}={ }^{\prime} E_{\infty}$. By its construction, the differential ' $d_{2}$ is a composite of two connecting maps

$$
H^{q}(\mathcal{Q}(\ell)) \stackrel{\alpha_{q}}{\longrightarrow} H^{q+1}\left(\mathcal{O}_{X}(\ell)\right) \stackrel{\beta_{q}}{\longrightarrow} H^{q+2}\left(\mathcal{I}_{X}(\ell)\right)
$$

coming from sequences (4-2), (4-3).
1. Assume $\ell \geq 0$, then the groups

$$
H^{\geq 1}\left(f_{*} \mathcal{O}_{\mathbb{P}^{2} \times \mathbb{P}^{2}} \otimes \mathcal{O}_{\mathbb{P}^{9}}(\ell)\right), \quad H^{\geq 1}\left(\mathcal{O}_{\mathbb{P}^{9}}(\ell)\right)
$$

vanish. Hence $\alpha_{q}$ is surjective for $q=0$ and an isomorphism for $q \geq 1$, whereas $\beta_{q}$ is always an isomorphism. Hence ${ }^{\prime} E_{3}^{p, q}=0$ unless $(p, q)=$ $(-5,0),(-5,1)$ or $(-4,0)$. Then

$$
\mathbb{H}^{-5}={ }^{\prime} E_{3}^{-5,0}={ }^{\prime} E_{2}^{-5,0}=H^{0}\left(\mathcal{I}_{X}(\ell)\right),
$$

and there is an extension

$$
0 \rightarrow{ }^{\prime} E_{3}^{-5,1} \rightarrow \mathbb{H}^{-4} \rightarrow{ }^{\prime} E_{3}^{-4,0} \rightarrow 0 .
$$

In fact ' $E_{3}^{-5,1}={ }^{\prime} E_{2}^{-5,1}=H^{1}\left(\mathcal{I}_{X}(\ell)\right)$ and ${ }^{\prime} E_{3}^{-4,0}$ is the kernel of the map $H^{0}(\mathcal{Q}(\ell)) \rightarrow H^{2}\left(\mathcal{I}_{X}(\ell)\right)$.

The terms $E_{1}^{p, q}$ are nonzero only for $q=0$, so $E_{2}=$ $E_{\infty}$ and $\mathbb{H}^{-5}=E_{2}^{-5,0}, \quad \mathbb{H}^{-4}=E_{2}^{-4,0}$.

2. Now assume $-9 \leq \ell \leq-1$, then the maps $\alpha_{q}, \beta_{q}$ are bijective for $0 \leq q \leq 2$. The only nonzero ' $E_{2}^{-4, q}$ term is at $q=2$, hence

$$
H^{4}\left(\mathcal{I}_{X}(\ell)\right)=H^{2}(\mathcal{Q}(\ell))=S_{-(3 \ell+1),-(3 \ell+2)} .
$$

If $\ell=-2,-1$, then each $E_{1}^{p, q}$ term is zero, hence $\mathbb{H}^{\bullet}$ is identically zero. If $\ell \leq-3$, then $E_{1}^{p, q}$ is nonzero exactly when $(p, q)=(\ell,-\ell)$. In that case, we have

$$
\mathbb{H}^{0}=S_{-(2 \ell+3)}^{*} \otimes S_{-(\ell+3)}^{*}, \quad \mathbb{H}^{j}=0 \quad \text { if } j \neq 0 .
$$

The preparations are over, and the computation proper may begin.

\subsection{Step V}

The procedure is to use formula $(2-7)$ on complexes $\left\{\check{E}_{1}^{p, q}\right\}_{p}$ and $\left\{E_{1}^{p, q}\right\}_{p}$ coming from the rows of $(2-6)$ and $(4-7)$. As explained earlier, if $\ell>0$, then $\left[E_{1}^{\bullet, 0}\right]$ can be calculated explicitly using plethysms followed by the L-R rule.

Since $M_{30}$ is a 20-dimensional summand of $S_{3}\left(S_{3}\right)$, it is necessarily $\{33,30\}$. Let $\ell=4$, then

$$
\begin{aligned}
{\left[\mathbb{H}^{-4}\right]-\left[\mathbb{H}^{-5}\right] } & =\left[E_{1}^{\bullet, 0}\right] \\
& =\sigma_{11,1}-\left(\sigma_{66}+\sigma_{63}+\sigma_{60}+\sigma_{51}+\sigma_{42}+\sigma_{00}\right) .
\end{aligned}
$$

(This is a heavy Maple computation.) The only nonzero terms in $(2-6)$ are $\check{E}_{1}^{0,0}$ and $\check{E}_{1}^{-1,0}$. Hence $H^{j}\left(\mathcal{I}_{X}(4)\right)=0$ for $j \neq 0$ and

$$
\left[H^{0}\left(\mathcal{I}_{X}(4)\right)\right]=\left[M_{40} \oplus\left(M_{30} \otimes S_{3}\right)\right]-\left[M_{31}\right] .
$$


From (4-10) and (4-11),

$$
\left[\mathbb{H}^{-5}\right]=\left[H^{0}\left(\mathcal{I}_{X}(4)\right)\right], \quad\left[\mathbb{H}^{-4}\right]=\left[H^{0}(\mathcal{Q}(4))\right]=\sigma_{11,1} .
$$

From $\left[M_{30}\right]=\sigma_{33}+\sigma_{30}$, the value of $\left[M_{30} \otimes S_{3}\right]$ is known by the L-R rule. From (4-14)-(4-16), we have

$$
\left[M_{40}\right]-\left[M_{31}\right]=\sigma_{66}-\left(\sigma_{42}+\sigma_{33}+\sigma_{21}\right) .
$$

Since $\operatorname{dim} M_{40}=28$, this forces $M_{40}=\{66\}$ and $M_{31}=$ $\{42,33,21\}$.

Now let $\ell=5$. Then (by an even heavier computation)

$$
\begin{aligned}
{\left[\mathbb{H}^{-4}\right]-\left[\mathbb{H}^{-5}\right]=} & {\left[E_{1}^{\bullet, 0}\right] } \\
= & \sigma_{14,1}-\left(\sigma_{96}+\sigma_{93}+\sigma_{90}+\sigma_{81}+\sigma_{75}\right. \\
& \left.+2 \sigma_{72}+\sigma_{63}+\sigma_{54}+\sigma_{51}+\sigma_{33}+\sigma_{30}\right) .
\end{aligned}
$$

From (2-6), we deduce that $\mathcal{I}_{X}(5)$ has no higher cohomology and

$$
\begin{aligned}
& {\left[H^{0}\left(\mathcal{I}_{X}(5)\right)\right]=\left[\check{E}_{1}^{0,0}\right]-\left[\check{E}_{1}^{-1,0}\right]+\left[\check{E}_{1}^{-2,0}\right]=} \\
& {\left[M_{30} \otimes S_{2}\left(S_{3}\right)\right]+\left[M_{40} \otimes S_{3}\right]-\left[M_{31} \otimes S_{3}\right]-\left[M_{41}\right]+\left[M_{32}\right] .}
\end{aligned}
$$

As before

$$
\left[\mathbb{H}^{-5}\right]=\left[H^{0}\left(\mathcal{I}_{X}(5)\right)\right], \quad\left[\mathbb{H}^{-4}\right]=\left[H^{0}(\mathcal{Q}(5))\right]=\sigma_{14,1} .
$$

Combining (4-17)-(4-19), we have a relation

$$
\left[M_{32}\right]+\left(\sigma_{75}+\sigma_{54}+\sigma_{33}\right)=\left[M_{41}\right]+\left(\sigma_{42}+\sigma_{21}+\sigma_{00}\right),
$$

which implies $M_{32}=\{42,21,00\}$ and $M_{41}=\{75,54,33\}$. Evidently we could continue the procedure with larger and larger $\ell$, and calculate all the $M_{j,-p}$. Alternately, we can twist by a negative $\ell$ and work our way from the other end of the resolution.

For instance, let $\ell=-1$. The only nontrivial map in $(2-6)$ is

$$
\check{E}_{1}^{-6,9} \longrightarrow \check{E}_{1}^{-5,9}, \quad \text { i.e., } \quad M_{46} \otimes S_{3}^{*} \longrightarrow M_{45} .
$$

It has kernel $H^{3}\left(\mathcal{I}_{X}(-1)\right)=H^{1}(\mathcal{Q}(-1))=0$, and cokernel $H^{4}\left(\mathcal{I}_{X}(-1)\right)=H^{2}(\mathcal{Q}(-1))=S_{21}$. Hence

$$
\sigma_{21}=\left[M_{45}\right]-\left[M_{46} \otimes S_{3}^{*}\right] .
$$

Substituting $M_{46}=\{00\}$, we have $M_{45}=\{33,21\}$. Now let $\ell=-2$ to calculate $M_{44}$, etc. These are the fruits of our labour:

$$
\begin{aligned}
& M_{30}=\{33,30\}, \\
& M_{31}=\{42,33,21\}, \\
& M_{32}=\{42,21,00\}, \\
& M_{33}=\{30\},
\end{aligned}
$$

$$
\begin{aligned}
& M_{40}=\{66\}, \\
& M_{41}=\{75,54,33\}, \\
& M_{42}=\{75,63,54,42,33,21\}, \\
& M_{43}=\{66,63,54,42,42,30,21,00\}, \\
& M_{44}=\{54,42,33,30,21\}, \\
& M_{45}=\{33,21\}, \\
& M_{46}=\{00\} .
\end{aligned}
$$

Scholium 4.4. In principle, we can work with negative $\ell$ throughout and bypass steps II-IV altogether. I see two reasons dictating against this. Firstly, the procedure becomes impractical as we move rightwards in the resolution. (For instance, in order to reach $M_{40}$, we need to set $\ell=-6$ and then calculate $M_{45} \otimes S_{5}\left(S_{3}^{*}\right)$ amongst other things.) Secondly, (and what is more to the point) I believe that the construction used here is of interest not confined to this example. In [Chipalkatti 01], it was used on varieties defined by binary forms having roots of specified multiplicities.

\section{THE LOCUS $X_{\Delta}$}

\begin{tabular}{c|ccccccccc} 
& 0 & 1 & 2 & 3 & 4 & 5 & 6 & 7 & 8 \\
\hline 4 & 35 & 119 & 210 & 252 & 210 & 120 & 45 & 10 & 1 \\
5 & & & 1 & & & & & &
\end{tabular}

The symmetric group $\mathfrak{S}_{3}$ acts on $\left(\mathbb{P} V^{*}\right)^{3}$ by permuting the factors, and $X_{\Delta}$ is the categorial quotient $\left(\mathbb{P} V^{*}\right)^{3} / \mathfrak{S}_{3}$. Hence for $\ell \geq 0$, the group $H^{0}\left(\mathcal{O}_{X}(\ell)\right)$ is the $\mathfrak{S}_{3}$-invariant part of

$$
\left(H^{0}\left(\mathbb{P} V^{*}, \mathcal{O}_{\mathbb{P}}(\ell)\right)\right)^{\otimes 3}=\left(S_{\ell}\right)^{\otimes 3},
$$

which is $S_{3}\left(S_{\ell}\right)$. By the same argument, $H^{j}\left(\mathcal{O}_{X}(\ell)\right)=0$ for $\ell \in \mathbf{Z}, 0<j<6$; and $H^{6}\left(\mathcal{O}_{X}(\ell)\right)=S_{3}\left(S_{-(\ell+3)}^{*}\right)$ for $\ell \leq-3$. Now we have identifications

$$
\begin{aligned}
0 \longrightarrow H^{0}\left(\mathcal{I}_{X}(\ell)\right) \longrightarrow H^{0}\left(\mathbb{P}^{9}, \mathcal{O}_{\mathbb{P}}(\ell)\right) & \longrightarrow H^{0}\left(X, \mathcal{O}_{X}(\ell)\right) \\
S_{\ell}\left(S_{3}\right) & \stackrel{\gamma}{\longrightarrow} S_{3}\left(S_{\ell}\right)
\end{aligned}
$$

If $\ell \geq 3$, then in (2-6) we have $\check{E}_{1}^{p, q}=0$ for $q>0$, so $H^{1}\left(\mathcal{I}_{X}(\ell)\right)=0$. Thus $\gamma$ is surjective for $\ell \geq 3$. (This is a rather special instance of Foulkes's conjecture-see e.g. [MacDonald 95, p.141].) Now formula (3-2) holds, and the calculation proceeds as in that case. This gets tedious for high values of $\ell$, but one can bypass it by calculating from the other end of the resolution.

For example, let $\ell=1$. The only nontrivial map in $(2-6)$ is

$\check{E}_{1}^{-8,9} \stackrel{d_{1}}{\longrightarrow} \check{E}_{1}^{-7,9}, \quad$ i.e., $\quad M_{48} \otimes H^{9}\left(\mathcal{O}_{\mathbb{P}}(-11)\right) \longrightarrow M_{47}$. 
Since $H^{j}\left(\mathbb{P}^{9}, \mathcal{I}_{X}(1)\right)=0$ for $j=1,2$, this map is an isomorphism. Hence $M_{47}=\{33\}$. Now let $\ell=0$ and calculate $M_{46}$, etc. The outcome is

$$
\begin{aligned}
& M_{40}=\{51\}, \\
& M_{41}=\{63,60,42\}, \\
& M_{42}=\{72,66,63,42,30\}, \\
& M_{43}=\{75,72,54,51,33,30\}, \\
& M_{44}=\{75,63,60,42,33\}, \\
& M_{45}=\{66,63,42,00\}, \\
& M_{46}=\{54,30\}, \\
& M_{47}=\{33\}, \\
& M_{48}=\{00\}, \\
& M_{52}=\{00\} .
\end{aligned}
$$

In [Salmon 1879, §226], Salmon gives a system of fortyfive equations each of degree 4 in the $\underline{a}$, which is satisfied iff the cubic $F$ breaks up into three lines. Thus the system generates an ideal $J$ such that $\sqrt{J}=I_{X}$. His construction is natural with respect to the $S L_{3}$-action, hence $(J)_{4} \subseteq M_{40}$ is a subrepresentation. Since $M_{40}$ is irreducible, $J=I_{X}$. It follows that there are ten dependencies in his system, something which is not a priori evident.

\section{Problem 5.1.}

1. The resolution shows some measure of duality, viz., $M_{42}=M_{44}^{*}, M_{41} \oplus \mathbf{C}=M_{45}^{*}, M_{43}=M_{43}^{*}$. There ought to be a theoretical explanation.

2. It follows from simple degree considerations that $M_{40}$ must be identical to the set of Brill's equations ([Gelfand et al. 94, p.139 ff]). It would be worthwhile to check this by direct calculation.

The next two computations are less conceptual that the preceding ones, in that they rely upon incidental features of the resolution.

\section{THE LOCUS $\boldsymbol{X}_{\varnothing}$}

\begin{tabular}{c|cccc} 
& 0 & 1 & 2 & 3 \\
\hline 8 & 35 & 70 & 45 & 8 \\
9 & & & & 1
\end{tabular}

We decompose $S_{8}\left(S_{3}\right)$ and look for a 35 -dimensional subrepresentation. The only possibilities for $M_{80}$ are found to be $\{51\}$ or $\{54\}$. In fact, it turns out that $M_{80}=\{54\}$. Let us grant this for the moment and postpone the justification to $§ 9.4$.
Substitute $\ell=9$ in (2-6). The only nontrivial map $\check{E}_{1}^{-1,0} \stackrel{d_{1}}{\longrightarrow} \check{E}_{1}^{0,0}$, i.e., $M_{81} \rightarrow M_{80} \otimes S_{3}$, is necessarily injective. On dimensional grounds $M_{81}=\{54,42,21\}$. Now $H^{6}\left(\mathcal{O}_{X}\right)=0$ (this was verified in Macaulay-2), hence $H^{7}\left(\mathcal{I}_{X}\right)=0$. But from (2-6), this group is the cokernel of the map

$$
\left(M_{83} \otimes S_{3}^{*}\right) \oplus S_{2}\left(S_{3}^{*}\right) \longrightarrow M_{82} .
$$

Since $S_{2}\left(S_{33}\right)=\{66,42\}$, it cannot by itself contribute a 45-dimensional summand. But then $M_{83}=$ $\{21\}$ is forced. Now the only possibilities for $M_{82}$ are $\{54,33\},\{42,33,21\}$. Since the resolution is minimal, the map $M_{93} \otimes R(-12) \longrightarrow M_{82} \otimes R(-10)$ is nonzero. Hence $M_{82} \otimes R_{2}=M_{82} \otimes S_{2}\left(S_{3}\right)$ must contain a trivial summand. This rules out $\{54,33\}$, so $M_{82}=\{42,33,21\}$. In sum,

$$
\begin{aligned}
& M_{80}=\{54\}, \\
& M_{81}=\{54,42,21\}, \\
& M_{82}=\{42,33,21\}, \\
& M_{83}=\{21\}, \\
& M_{93}=\{00\} .
\end{aligned}
$$

In [Salmon 1879, §240], Salmon gives a system of equations each of degree 8 in the $\underline{a}$, which is satisfied iff the cubic $F$ is factorisable. Using an argument similar to the case $X_{\Delta}$, it follows that his system generates $I_{X_{\varnothing}}$.

Problem 6.1. Define $\mathcal{Q}=\operatorname{coker}\left(\mathcal{O}_{X_{\varnothing}} \rightarrow f_{*} \mathcal{O}_{\mathbb{P} S_{2}^{*} \times \mathbb{P} V^{*}}\right)$ as in the case of $X_{\neq}$. It would be of interest to calculate it explicitly along the lines of lemma 4.1, or even to calculate the groups $H^{\bullet}(\mathcal{Q}(\ell))$. Once this is done, there is no difficulty in adapting steps II-V to $X_{\varnothing}$.

\section{THE LOCUS $\boldsymbol{X}_{\mid \mathrm{O}}$}

\begin{tabular}{c|ccc} 
& 0 & 1 & 2 \\
\hline 4 & 1 & & \\
5 & 10 & 8 & \\
6 & & 10 & 8
\end{tabular}

Now $M_{40}=\{00\}$ and $H^{0}\left(\mathcal{I}_{X}(5)\right)=\left(M_{40} \otimes S_{3}\right) \oplus M_{50}$ is a 20 -dimensional module inside $H^{0}\left(\mathcal{O}_{\mathbb{P}}(5)\right)=S_{5}\left(S_{3}\right)$. Decomposing the latter, on dimensional grounds the module can only be $\{33,30\}$. So $M_{50}=\{33\}$.

Now $M_{51}$ is a summand of $\left(M_{40} \otimes R_{2}\right) \oplus\left(M_{50} \otimes R_{1}\right)$, so it must be $\{21\}$. Since $M_{61}$ is a summand of $\left(M_{40} \otimes R_{3}\right) \oplus$ $\left(M_{50} \otimes R_{2}\right)$, it is either $\{33\}$ or $\{30\}$, we do not yet know which. Decompose $\left(M_{61} \otimes R_{1}\right) \oplus\left(M_{51} \otimes R_{2}\right)$ for each 
of the possibilities, and one sees that $M_{62}$ is necessarily $\{21\}$.

It remains to determine $M_{61}$. Since $X$ is 6dimensional, $H^{8}\left(\mathcal{I}_{X}(-3)\right)=0$. Substituting $\ell=-3$ in (2-6), this group appears as the cokernel of

$$
M_{62} \otimes H^{9}\left(\mathbb{P}^{9}, \mathcal{O}_{\mathbb{P}}(-11)\right) \longrightarrow M_{61} .
$$

The only 10-dimensional summand of $\{21\} \otimes\{33\}$ is $\{33\}$, so $M_{61}=\{33\}$. Thus

$$
\begin{aligned}
& M_{40}=\{00\}, M_{50}=\{33\}, M_{51}=\{21\}, \\
& M_{61}=\{33\}, M_{62}=\{21\} .
\end{aligned}
$$

Problem 7.1. Without recourse to a machine computation, show that $X_{\mid \bigcirc}$ is arithmetically Cohen-Macaulay. (This will probably involve the theory of complete conics.)

\section{THE LOCUS $X_{Y}$}

\begin{tabular}{c|cccc} 
& 0 & 1 & 2 & 3 \\
\hline 3 & 20 & 45 & 36 & 10
\end{tabular}

This is a rank variety in the sense of Porras [Porras 96], and its resolution is deduced there (Prop. 4.2.3). We summarise the solution.

A subspace $W$ of $V^{*}$ gives an inclusion $\mathbb{P}\left(S_{3} W\right) \subseteq$ $\mathbb{P}\left(S_{3} V^{*}\right)$. Then

$X_{Y}=\left\{F: F \in \mathbb{P}\left(S_{3} W\right)\right.$ for some proper subspace $\left.W\right\}$.

(This is so, because the $L_{i}$ define concurrent lines iff they fail to span $V^{*}$.) The multiplication $S_{2} \otimes S_{1} \longrightarrow S_{3}$ gives a map of vector bundles

$$
\alpha: \underbrace{S_{2} \otimes \mathcal{O}_{\mathbb{P}}(-1)}_{\mathcal{B}} \longrightarrow V^{*} \otimes \mathcal{O}_{\mathbb{P}}
$$

on $\mathbb{P} S_{3}^{*}$. Then $X$ coincides (as a scheme) with the degeneracy locus $\{\operatorname{rank} \alpha \leq 2\}$, and the Eagon-Northcott complex of $\alpha$ resolves its structure sheaf.

$$
\begin{aligned}
& 0 \rightarrow \wedge^{6} \underset{\otimes S}{\underset{\otimes}{3}} \rightarrow \wedge^{5} \underset{\otimes S}{\underset{\otimes}{2}} \rightarrow \wedge^{4} \underset{\otimes S}{\underset{\otimes}{\longrightarrow}} \\
& \rightarrow \wedge^{3} \underset{\rightarrow \mathcal{O}}{\longrightarrow} \rightarrow \mathcal{O}_{X_{Y}} \rightarrow 0 .
\end{aligned}
$$
Now $\wedge^{j} \underset{\text { further }}{\Rightarrow} S_{2} \otimes \mathcal{O}_{\mathbb{P}}(-j)$, and after decomposing these

$$
\begin{aligned}
& M_{30}=\{33,30\}, M_{31}=\{42,33,21\} \\
& M_{32}=\{42,21,00\}, M_{33}=\{30\} .
\end{aligned}
$$

\section{IDEAL GENERATORS AND CONCOMITANTS}

Let $X$ be any of the loci above. For $\ell \geq 1$, we have an exact sequence

$$
\left(I_{X}\right)_{\ell-1} \otimes S_{3} \longrightarrow S_{\ell}\left(S_{3}\right) \stackrel{g_{\ell}}{\longrightarrow} U_{\ell} \longrightarrow 0 .
$$

The module $M_{\ell, 0}$ consists of degree $\ell$ primitive generators for $I_{X}$, as such it is a submodule of $U_{\ell}$. Let $S_{m+n, n} \subseteq$ $M_{\ell, 0}$ be a direct summand. By choosing an equivariant splitting of $g_{\ell}$, we may write $S_{m+n, n} \subseteq S_{\ell}\left(S_{3}\right)$. Then by $\S 2.2$, to specify this inclusion is to specify a concomitant $\Phi_{\ell, m, n}$.

It turns out that in every case under consideration, there is only one copy of $S_{m+n, n}$ inside $S_{\ell}\left(S_{3}\right)$, hence the inclusion is independent of the choice of the splitting. ${ }^{5}$ Thus it is enough to produce some symbolic expression of type $(\ell, m, n)$ (by trial and error), and to verify that it does not vanish identically after substitution. Then it must be the $\Phi_{\ell, m, n}$ that we are looking for. Besides doing this, in each case I have verified by direct computation (in Macaulay-2) that $\Phi$ vanishes on $X$. Although logically superfluous, this is a very useful check against the propagation of error.

\subsection{Case $X_{\equiv}$}

Corresponding to $M_{20}=\{42\}$, we need a concomitant of type $(2,2,2)$. The only legal symbolic expression is

$$
\Phi_{222}=(\alpha \beta u)^{2} \alpha_{x} \beta_{x} .
$$

To recapitulate, let $T_{1}, \ldots, T_{27}$ be the semistandard tableaux on $(4,2)$ (see $\S 2.1)$ and write $\Phi_{222}=\sum_{i=1}^{27} f_{i} \otimes X_{T_{i}}$. (A priori, after expansion $\Phi$ will contain nonstandard monomials such as $x_{1}^{2} u_{1} u_{3}$. These can be rewritten as linear combinations of the $X_{T}$ using the straightening law-see [Fulton 97, §8.1].) Then $I_{X}=\left(f_{1}, \ldots, f_{27}\right)$. This can be rephrased as a criterion:

A ternary cubic $F$ can be written as the cube of a linear form iff the concomitant $(\alpha \beta u)^{2} \alpha_{x} \beta_{x}$ vanishes on $F$. There is a similar claim for each $X$.

\subsection{Case $X_{\neq}$}

$$
\begin{aligned}
M_{30} \longmapsto \Phi_{303} & =(\alpha \beta \gamma)(\alpha \beta u)(\alpha \gamma u)(\beta \gamma u), \\
\Phi_{330} & =(\alpha \beta \gamma)^{2} \alpha_{x} \beta_{x} \gamma_{x} .
\end{aligned}
$$

These are respectively the Cayleyan and the Hessian of $F$ (see [Salmon 1879, §218,219]).

$$
M_{40} \longmapsto \Phi_{406}=(\alpha \beta u)^{2}(\gamma \delta u)^{2}(\alpha \delta u)(\beta \gamma u) .
$$

\footnotetext{
${ }^{5}$ There is no difficulty in adapting to the case when this is
not so. not so.
} 


\subsection{Case $X_{\Delta}$}

$$
M_{40} \longmapsto \Phi_{441}=(\alpha \beta \gamma)(\alpha \gamma \delta)(\alpha \beta u) \beta_{x} \gamma_{x} \delta_{x}^{2} .
$$

\subsection{Case $\boldsymbol{X}_{\varnothing}$}

We are yet to prove that $M_{80}$ is $\{54\}$ and not $\{51\}$. There is a well known invariant of ternary cubics in degree 4, namely the Aronhold invariant (see [Sturmfels 93, p. 167])

$$
\Phi_{400}=(\alpha \beta \gamma)(\alpha \beta \delta)(\alpha \gamma \delta)(\beta \gamma \delta) .
$$

Decomposing $S_{8}\left(S_{3}\right)$, one sees that it houses exactly one copy each of $S_{51}, S_{54}$. Hence there is one concomitant each of type $(8,4,1),(8,1,4)$. Now $\Phi_{841}$ is necessarily equal to the product $\Phi_{400} \Phi_{441}$. Since $I_{X_{\varnothing}}$ has no generators in degree 4 , none of the factors can vanish on $X_{\varnothing}$. Hence $M_{80} \neq\{51\}$. Thus

$$
\begin{aligned}
M_{80} & \longmapsto \Phi_{814} \\
& =\alpha_{x}(\alpha \beta \gamma)(\alpha \beta \delta)(\beta \gamma \epsilon)(\gamma \zeta u)(\delta \epsilon u)(\delta \eta u)(\epsilon \theta u)(\zeta \eta \theta)^{2} .
\end{aligned}
$$

It is quite easy to concoct symbolic expressions of type $(8,1,4)$, but most will vanish identically after expansion. It cost the author a week's labour to find one which does not.

\subsection{Case $\boldsymbol{X}_{\mid \mathrm{O}}$}

$$
\begin{aligned}
M_{40} \longmapsto \Phi_{400}(\text { as written above). } & \\
M_{50} \longmapsto \Phi_{503} & =(\alpha \beta \gamma)(\alpha \beta \delta)(\beta \gamma \epsilon)(\alpha \gamma u)(\delta \epsilon u)^{2} .
\end{aligned}
$$

\subsection{Case $\mathrm{X}_{\mathrm{Y}}$}

$$
M_{30} \longmapsto \Phi_{303}, \Phi_{330} \text { (as written above). }
$$

In fact, the Hessian vanishes identically iff the Cayleyan does. (This is an easy deduction from the formulae in [Cayley 1881].) Hence either one defines the locus settheoretically. Also see [Olver 99, p. 234] for a discussion of the Gordan-Nöther theorem.

Scholium 9.1. The symbolic method can be used for describing the higher syzygies as well. We will give an illustration of this idea but will not attempt any systematic treatment. Consider the resolution of $X_{\equiv}$, in particular the submodule $S_{54} \subseteq M_{21}$. Keep the notation of $\S 9.1$. We have the differential

$$
S_{54} \otimes \mathcal{O}_{\mathbb{P}}(-3) \stackrel{\partial^{1}}{\longrightarrow} S_{42} \otimes \mathcal{O}_{\mathbb{P}}(-2),
$$

or equivalently, a map

$$
\mathcal{O}_{\mathbb{P}} \longrightarrow S_{42} \otimes S_{51} \otimes \mathcal{O}_{\mathbb{P}}(1),
$$

or equivalently, an element

$$
\Psi \in H^{0}\left(\mathbb{P}^{9}, S_{42} \otimes S_{51} \otimes \mathcal{O}_{\mathbb{P}}(1)\right)=S_{42} \otimes S_{51} \otimes S_{3} .
$$

We would like to represent $\Psi$ symbolically. Let $\underline{y}=$ $\left\{y_{1}, y_{2}, y_{3}\right\}$ be 'copies' of the variables $\left\{x_{1}, x_{2}, x_{3}\right\}$, and ditto for $\underline{v}=\left\{v_{1}, v_{2}, v_{3}\right\}$ answering to $\left\{u_{1}, u_{2}, u_{3}\right\}$. Let $\mathbb{T}_{1}, \ldots, \mathbb{T}_{35}$ be the semistandard tableaux on numbers $1,2,3$ on the Young diagram of $(5,1)$. Exactly as in $\S 2.1$, $Y_{\mathbb{T}_{j}}$ will denote a monomial in the $\underline{y}, \underline{v}$ corresponding to $\mathbb{T}_{j}$.

Write $\alpha_{y}=\alpha_{1} y_{1}+\alpha_{2} y_{2}+\alpha_{3} y_{3}, v_{x}=v_{1} x_{1}+v_{2} x_{2}+v_{3} x_{3}$, and

$$
(\alpha u v)=\left|\begin{array}{lll}
\alpha_{1} & \alpha_{2} & \alpha_{3} \\
u_{1} & u_{2} & u_{3} \\
v_{1} & v_{2} & v_{3}
\end{array}\right| .
$$

A calculation (which we suppress) shows that $\Psi=$ $(\alpha u v)^{2} \alpha_{y} v_{x}^{2}$. That is to say, after expanding the product and substituting, we can write

$$
\Psi=\sum_{j=1}^{35}\left(\sum_{i=1}^{27} h_{i j} \otimes X_{T_{i}}\right) \otimes Y_{\mathbb{T}_{j}},
$$

where $h_{i j}$ are linear forms in the $\underline{a}$. Let $\Sigma_{j}$ be the sum in the parentheses multiplying $Y_{\mathbb{T}_{j}}$. Then $\Sigma_{j}$ represents a linear syzygy between the ideal generators $f_{i}$, namely $\sum_{i=1}^{27} h_{i j} f_{i}=0$. This accounts for the 35 syzygies corresponding to the summand $S_{54} \subseteq M_{21}$, and of course other summands are given by other symbolic expressions. In fact

$$
\begin{aligned}
& S_{51} \longmapsto \alpha_{x} \alpha_{y}^{2} v_{x} u_{y}^{2}, \quad S_{42} \longmapsto(\alpha u v) \alpha_{x} \alpha_{y} v_{x} u_{y}, \\
& S_{21} \longmapsto(\alpha u v) \alpha_{x}^{2} u_{y} .
\end{aligned}
$$

The complete system of concomitants for a ternary cubic is given by Cayley [Cayley 1881]. I enclose a table collating our notation with his.

$$
\begin{array}{llllll}
\Phi_{222}=\Theta & (\text { no.4), } & \Phi_{303}=P & (\text { no. }), & \Phi_{330}=H & (\text { no.12) }, \\
\Phi_{406}=F & (\text { no.17), } & \Phi_{441}=J & (\text { no. } 19), & \Phi_{400}=S & (\text { no.1 }), \\
\Phi_{814}=\bar{J} & (\text { no. } 27), & \Phi_{503}=Q & (\text { no. } 16) . & &
\end{array}
$$

Scholium 9.2. The invariant $S$ is ubiquitous in the geometry of plane cubics, it is essentially the same as the Eisenstein series $g_{2}$. There is a sextic invariant (also called $T$ )

$$
\Phi_{600}=(\alpha \beta \gamma)(\alpha \beta \delta)(\beta \gamma \epsilon)(\alpha \gamma \zeta)(\delta \epsilon \zeta)^{2},
$$

which is $g_{3}$ in a different guise. The discriminant $\Delta$ of $F$ is a linear combination of $S^{3}$ and $T^{2}$ (the coefficients depend on one's normalisation). Now $\Delta=0$ is the closure 
of the locus of nodal cubics. The locus of cuspidal cubics is the complete intersection $S=T=0$. (See [Salmon $1879, \S 224]$.)

Several geometric theorems about the Cayleyan can be found in [Cayley 1856] (where it is called the Pippian). The invariant $S$ vanishes on a smooth cubic curve iff the latter is projectively isomorphic to the Fermat cubic $x_{1}^{3}+$ $x_{2}^{3}+x_{3}^{3}=0$. Such cubics are called anharmonic. See [Dolgachev and Kanev 93] for some beautiful results in this vein.

\section{REFERENCES}

[Carrell and Dieudonné 71] J. Carrell and J. Dieudonné. Invariant theory, old and new. Academic Press, New YorkLondon, 1971.

[Cayley 1856] A. Cayley. "A memoir on curves of the third order." Philosophical Transactions of the Royal Society of London. cxlvii (1856), 415-446. Reprinted in Volume II of his Collected Mathematical Papers, Cambridge Univ. Press, 1889.

[Cayley 1881] A. Cayley. "On the 34 concomitants of the ternary cubic." American J. of Math. iv (1881), 1-15. Reprinted in Volume XI of his Collected Mathematical Papers, Cambridge Univ. Press, 1889.

[Chipalkatti 00] J. Chipalkatti. "A generalization of Castelnuovo regularity to Grassmann varieties." Manuscripta Math. 102 (2000), 447-464.

[Chipalkatti 01] J. Chipalkatti. "On equations defining coincident root loci." Preprint, 2001. (Available from the Algebraic Geometry archives at http://xxx.lanl.gov)

[Dolgachev and Kanev 93] I. Dolgachev and V. Kanev. "Polar covariants of cubics and quartics." Adv. in Math. 98:2 (1993), 216-301.

[Fulton 97] W. Fulton. Young Tableaux. London Mathematical Society Student Texts. Cambridge Univ. Press, Cambridge, 1997.

[Fulton and Harris 91] W. Fulton and J. Harris. Representation Theory, A First Course. Graduate Texts in Mathematics. Springer-Verlag, New York, 1991.
[Gelfand et al. 94] I. M. Gelfand, M. M. Kapranov, and A. V. Zelevinsky. Discriminants, Resultants and Multidimensional Determinants. Birkhäuser, Boston, 1994.

[Grace and Young 1903] J. H. Grace and A. Young. The algebra of invariants. 1903. Reprinted by Chelsea, New York in 1982.

[Green 84] M. Green. "Koszul cohomology and the geometry of projective varieties, II." J. Diff. Geometry, 20:1 (1984) 279-289.

[Harris 92] J. Harris. Algebraic Geometry, A First Course. Graduate Texts in Mathematics. Springer-Verlag, New York, 1992.

[Hartshorne 77] R. Hartshorne. Algebraic Geometry. Graduate Texts in Mathematics. Springer-Verlag, New York, 1977.

[Kraft 85] H. Kraft. Geometrische Methoden in der Invariantentheorie. Aspekte der Mathematik. Vieweg, Braunschweig, 1985.

[MacDonald 95] I. G. MacDonald. Symmetric Functions and Hall Polynomials, 2nd edition. Oxford University Press, Oxford, 1995.

[Olver 99] P. Olver. Classical Invariant Theory. London Mathematical Society Student Texts. Cambridge Univ. Press, Cambridge, 1999.

[Porras 96] O. Porras. "Rank varieties and their resolutions." J. of Algebra, 186:3 (1996), 677-723.

[Salmon 1876] G. Salmon. Higher algebra, 5th ed., 1876. Reprinted by Chelsea, New York in 1964.

[Salmon 1879] G. Salmon. Higher plane curves, 3rd ed., 1879. Reprinted by Chelsea, New York in 1960.

[Sturmfels 93] B. Sturmfels. Algorithms in Invariant Theory. Texts and Monographs in Symbolic Computation. Springer-Verlag, Wien-New York, 1993.

[Weitzenböck 1923] H. Weitzenböck. Invarianten-Theorie. Noordhoff, 1923.

[Weyl 1939] H. Weyl. Classical Groups. Their Invariants and Representations. Princeton Univ. Press, Princeton, 1939.

Jaydeep V. Chipalkatti, Department of Mathematics and Statistics, 416 Jeffery Hall, Queen's University, Kingston, ON K7L 3N6, Canada (jaydeep@mast.queensu.ca)

Received April 26, 2001; accepted in revised form December 27, 2001. 